\title{
The immune imbalance of Treg/Th17 in secretory otitis media patients may be related to PI3K/Akt/mTOR signaling activation in the middle ear mucosa
}

\author{
Zhiyuan Tang ${ }^{1}$, Xianhai Zeng ${ }^{1}$, Juanjuan $\mathrm{Li}^{1}$, Hua Zhang ${ }^{1}$, Hancheng $\mathrm{Wu}^{2}$, Suqing \\ Zhuang $^{2}$, and Yiqing Zheng ${ }^{3}$ \\ ${ }^{1}$ Shenzhen Longgang E.N.T hospital \& Shenzhen Key Laboratory of E.N.T, Institute of \\ E.N.T \\ ${ }^{2}$ Shenzhen Longgang E.N.T hospital \& Shenzhen Key Laboratory of E.N.T, Institute of \\ E.N.T \\ ${ }^{3}$ Sun Yat-Sen Memorial Hospital
}

September 16, 2020

\begin{abstract}
Objective: To assess whether secretory otitis media may be caused by immune imbalance of Treg/Th17 mediated by PI3K/Akt/mTOR signaling, so as to find new therapeutic target. Methods: IL-17, TGF- and IL-6, IL-10 and Th17 cytokines were detected in peripheral blood of OME patients (PC group) and healthy people (NC group) by ELISA. The expression of ROR $\mathrm{t}$ mRNA and Foxp3mRNA in PBMC was detected by RT-PCR. OME rat model was established and the changes of lymphocytes in middle ear mucosa and spleen and PI3K/Akt/mTOR signaling in middle ear mucosa were detected by HE staining, IHC, WB and flow cytometry. Results: The immune imbalance of Treg/Th17 in secretory otitis media (OME) was confirmed by the expression of cytokines in OME serum and analysis of ROR T and Foxp3 mRNA which was Th17 and Treg specific transcription respectively. OME rat model further confirmed that Treg/Th17 imbalance could lead to OME as demonstrated by staining of MIDDLE ear mucosa and expression of ROR T and Foxp3. PI3K, Akt, and mTOR proteins were expressed in the MIDDLE ear mucosa of OME group and CON group, respectively. Compared with CON group, the expression of P-MTOR and P-PI3K proteins in the middle ear mucosa of OME group was significantly increased. Conclusions: Treg/Th17 imbalances are found in OME patients and OME animal model and the pathogenic mechanism may be due to systemic abnormal immune response, activated $\mathrm{PI} 3 \mathrm{~K} / \mathrm{Akt} / \mathrm{mTOR}$ signaling, abnormal $\mathrm{T}$ cell differentiation, leading to middle ear mucosal hyperemia, edema and subsequent occurrence of OME.
\end{abstract}

\section{Introduction}

Otitis Media of Effusion (OME) is a common multiple disease in E.N.T. Department, mainly manifested as Middle Ear Effusion (MEE) in the drum room. Generally, MEE can be diagnosed as OME after the Acute Otitis Media (AOM) lasts for at least 3 months or has been found to be asymptomatic during the specialist examination [1]. Allergy has been reported to increase the incidence of OME by 2-4.5 times compared with non-allergic people [2-3], and allergic inflammation of the upper respiratory mucosa may involve in OME pathogenesis [4], suggesting that there is a correlation between and allergy.

At present, the main causes of OME are mechanical obstruction of the eustaphylococcal tube, infection, and allergy, among which the mechanism of allergy is still debated. In recent years, according to the theory of 
"consistency of upper and lower respiratory tract inflammation", "all parts of upper and lower respiratory tract are anatomically continuous, and inflammation is rarely confined to one part. In recent years, studies on OME pathogenesis have found that innate immunity plays a protective role in the middle ear. When inflammation occurs, this protective response is destroyed, resulting in immune imbalance of the middle ear [5-6]. An increasing number of studies [7-8] have shown that activation of downstream inflammatory signaling pathways caused by cellular immunity and some humoral immune factors is a contributing factor to OME, and is related to immune factors affecting lymphatic reflux, promoting luminal mucosa swelling and gland secretion, and thus leading to OME. The mechanism of Th17/Treg cell-mediated immune response has become the focus of multidisciplinary research [8-9].However, few studies on Th17 and Treg cells in OME have been reported. In this study, the role of $\mathrm{T}$ cells in OME pathogenesis and its significance were explored from the perspective that the immune response induced by Treg/Th17 imbalance may be the cause of OME.

\section{Methods and materials}

\section{Testing of clinical samples}

Peripheral blood and middle ear effusion (MEE) from PATIENTS with OME (PC) and healthy volunteers (NC)

Repeatedly OME patients (PC Group) and obviously out of ear disease and systemic abnormal immune response to disease (after IgE and clinical symptom screening) of healthy people (NC Group) were recruited to collect $2 \mathrm{ml}$ venous blood. Signed informed consent was obtained from patients and healthy people and this study was approved by the ethnic committee of Shenzhen Longgang District Hospital of Otolaryngology (Shenzhen Institute of Otolaryngology).

Peripheral blood mononuclear cells (PBMC) from the remaining blood were isolated by density gradient centrifugation and RNA was extracted from the harvested cells using TRIzol according to the manufacturer's instructions. CDNA was synthesized by Sangon Biotech (Shanghai, China) and amplified by QRT-PCR (Applied Biosystems, Foster City, California, US). The data were analyzed using relative gene expression methods and the GAPDH Ct values in the samples were standardized. Each sample shall be measured in triplicate.

Primer sequences used for PCR amplification are as follows:

\begin{tabular}{ll}
\hline Foxp3 forward & GGGTAGCCATGGAAACAGCA \\
Foxp3 reverse & TCGCATGTTGTGGAACTTGAAGTAG \\
ROR $r$ forward & CTGCAAGACTCATCGCCAAAG \\
ROR rt reverse & TTTCCACATGCTGGCTACACA \\
GAPDH forward & CCTGCACCACCAACTGCTTA \\
GAPDH reverse & GGCCATCCACAGTCTTCTGAG \\
\hline
\end{tabular}

\section{Verification of OME animal models}

\subsection{Establishment of animal model}

The OME animal model was established according to a previous study by Hardy et al. [10], which were modified slightly, as follows:

Model group (OME Group) was intraperitoneally injected with 10mg Ovalbumin (Sigma-Aldrich, US) which was dissolved in $0.8 \mathrm{ml}$ PBS phosphate buffer $(0.01 \mathrm{M}$, pH7.4) plus $5.14 \mathrm{mg}$ aluminum hydroxide gel adjuvant, once a week for 2 weeks. 2 weeks after receiving chloramine $(100 \mathrm{mg} / \mathrm{ml})+$ stability $(5 \mathrm{mg} / \mathrm{ml})$ by intraperitoneal injection of $2: 1$ mixture $(3 \mathrm{ml} / \mathrm{kg})$ depth of anesthesia, the supine position, under the operating microscope is equal to the level of hyoid, a crosscutting was done for separation of hyoid scapula muscle and hyoid bone muscle to expose double ear, bubble bottom wall respectively, aseptic technique was used to trace syringe. OVA $2 \mathrm{mg}$ of $50 \mathrm{uL}$ PBS liquid was injected into bilateral listen to bubble, which is 
ear stimulating stage (before injection, about $2 \mathrm{~mm}$ hole was made from the injection site to balance the middle ear cavity injection pressure and prevents the tympanic membrane rupture). Gelatine sponge was inserted to prolong OVA duration. After injection, 2 small holes were sealed with bone wax, which was the intraaural stimulation stage. Control group (CON) was sensitized with PBS. Intramuscular injection of penicillin prevented bacterial infection before stimulation. After the operation, animals were sacrificed 2 days after feeding in cages in a clean environment. The researchers did not know the allocation of groups when evaluating the results. All animals were treated in accordance with the guidelines for the use of experimental animals which were approved by the Ethnic Committee of Shenzhen Longgang E.N.T Hospital (Shenzhen Otorhinolaryngology Institute).

\subsection{General observation of animals}

After anesthesia, the two eardrums of each group were observed under the ear endoscope, then the heads were severed, and bilateral auditory vesicles were removed rapidly. The mucous membrane and effusion in the drum chamber were observed under anatomical microscope.

\subsection{Detection of middle ear tissues}

The right auditory vesicles were isolated, fixed, decalcified and embedded according to conventional methods, and the expression of ROR T and FOXP3 was assessed by histological sections and immunohistochemical staining.

\subsection{Flow cytometry analysis}

PBMC was extracted from OME and CON rat spleen using rat lymphocyte separation solution (Solarbio Science, Beijing, China), and $\mathrm{T}$ cells were sorted by flow cytometry. To detect Treg cells, PBMC stained with eZFluorTM anti-human CD4-FITC and CD25-APC mixture, and incubated with an effective fixation/permeation solution. According to the manufacturer's instructions (Thermo Fisher, Waltham, Massachusetts, US), intracellular staining was performed with anti-human FOXp3-PE or rat IgG2a K isotype control PE. To evaluate Th17 cells, PBMC was stimulated for 5 hours in a complete medium (supplemented with 10\% FBS and $200 \mathrm{mM}$ L-GLN RPMI 1640) and then incubated with FITC, an anti-human CD4-FITC, or mouse IgG1 K isotype control, and then with an effective fixative/permeable solution and permeable buffer. According to the manufacturer (Thermo Fisher, Waltham, Massachusetts, US), intracellular staining was performed with anti-human IL-17A-PE or mouse IgG1 K isotype control PE. FACSCalibur flow cytometry (BD Biosciences, US) was used for analysis and CellQuest software (BD Biosciences, Franklin Lakes, NJ, US) analyzed data.

\subsection{Western blot}

The OME and CON groups of auditory vesicle middle ear mucosal tissues were homogenized on ice for protein extraction which was then quantified using BCA kit (Thermo Fisher, Waltham, Massachusetts, US) and standardized to $2 \mathrm{~g} / \mathrm{uL}$ protein density followed by western blot analysis. The ECL chemiluminescence detection system was used to visualize protein bands. The protein expression was standardized to GAPDH.

\section{Statistical analysis}

Data are expressed as the means \pm standard deviation. The SPSS 22.0 software was used to perform the statistical analyses of the data, and a single-factor analysis of variance was used to determine the significance of differences between groups.

\section{Results}

Treg/Th17 imbalance was found in peripheral blood and middle ear of OME patients (PC group) 
We collected middle ear effusion from 14 OME patients (PC) with underlying allergic disease and peripheral blood from 14 healthy volunteers (NC). The concentrations of TGF- /IL-10 and Th17 marker cytokine IL-17/IL-6 in serum were measured by ELISA. Compared with NC group, the level of IL-6 (IL$6: 18.79 \pm 3.35$ vs. $23.59 \pm .57 \mathrm{pg} / \mathrm{ml}$ ) and IL-17 (IL-17:7.62 \pm 1.96 vs. $1.51 \pm 0.47 \mathrm{pg} / \mathrm{ml}$ ) in PC group was significantly higher (P\&LT;0.001); However, level of IL-10 (1.63 \pm 0.38 vs. $3.93 \pm 0.43 \mathrm{pg} / \mathrm{ml})$ and TGF- $(17.94 \pm 3.01$ vs.62.04 $\pm 2.65 \mathrm{pg} / \mathrm{ml})$ was decreased in PC group $(\mathrm{P}<0.001)$. The levels of IL-6(45.95 \pm 5.18 vs.18.79 \pm 3.35$)$ and IL-17(18.61 \pm 1.21 vs.7.62 \pm 1.96$)$ in PC group were significantly higher than that of the peripheral serum.

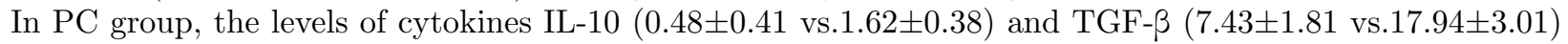
were decreased $(\mathrm{P}<0.001)$. The above results suggest that the pathogenesis of OME is not only the local allergy of the middle ear, but also the systemic allergy. The abnormal immune response affects the differentiation direction of $\mathrm{T}$ cells, resulting in imbalance of Treg/Th17 and finally the local stimulation in the middle ear, which may be one of the causes of OME.

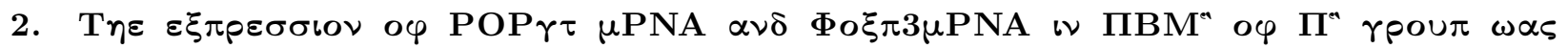

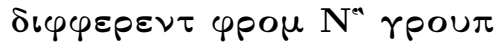

The expression of ROR $\gamma \mathrm{T}$ mRNA and Foxp3 mRNA in PC group and NC group were detected by PCR and showed increased levels in $\mathrm{PC}$ group $(\mathrm{P}<0.05)$, Foxp3 mRNA expression in $\mathrm{PC}$ group was lower than NC group $(\mathrm{P}<0.05)$. In addition, OME patients had systemic sensitization caused by Treg/Th17imbalance, which may create conditions for local excitation of the middle ear.

\section{Abnormal immune response inOME animal model;}

\subsection{Endoscopic and histological observation of the middle ear}

Under endoscopy, the eardrum of CON group was transparent without obvious effusion in the drum room. In OME group, the eardrum was orange, turbidized and thickened, with obvious effusion in the drum chamber (Figure 3). HE staining showed that the tympanum ciliated mucosal epithelium in the control group was thin, scattered in goblet cells, and there was no obvious inflammatory cell infiltration in the lamina propria. The mucosal layer of the experimental group was thickened, with local congestion, tissue edema, glands dilated, eosinophil aggregation of plasma cells, increased goblet cells and hyperchromatic nuclei (Figure 4). The above experiments demonstrate the success of OME modeling.

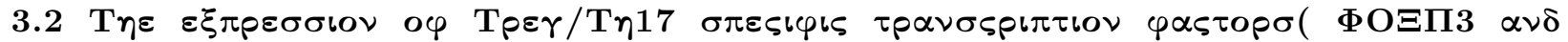 $\left.\operatorname{POP}_{\gamma} \tau\right)$ is $\varphi \mu \beta \alpha \lambda \alpha \nu \varsigma \varepsilon \delta$}

IHC staining showed that FOXP3 in OME and CON group middle ear mucosa were differentially expressed and ROR $r$ t was stained pale yellow with different expression in OME and CON group. OME group showed ROR $r t$ antigen expression in the cytoplasm and capsular, tan, sediment samples with higher positive cells rate than CON group. FOXP3 antigen was expressed in the cytoplasm and envelop of the middle ear mucous membrane in OME group and the positive cell rate was weaker than CON group.

\subsection{Flow cytometry detection revealed animbalance of Treg/Th17 in the OME animal model}

Flow cytometry detection revealed that the Treg proportion was $3.57 \pm 0.66 \%$ in OME group and $14.99 \pm 2.49 \%$ in CON group,. The proportion of Th17 in the OME group was $12.17 \pm 1.90 \%$, and the proportion of Treg in the CON group was $3.29 \pm 0.49 \%$. The above results indicated that the initial $\mathrm{T}$ cells in OME group showed a decreased tendency to differentiate into Treg and an increased tendency to differentiate into Th17. Further analysis found that Treg/Th17 ratio of CD4 T cells in OME group was decreased, while Th17 ratio of CD4 $\mathrm{T}$ cells was increased, and Treg/Th17 ratio was decreased, indicating Treg/Th17 imbalance in OME.

\subsection{OME animal model showed activated PI3K/Akt/mTOR signaling}

Western Blot detection of PI3K/Akt/mTOR pathway related protein expression (Figure 8) showed that compared with CON group, OME group had significantly increased p-mTOR and p-PI3K protein expression, suggesting that under the stimulation of allergens, phosphorylation of PI3K and mTOR occurs locally in 
the mucosa of the middle ear, and the PI3K/Akt/mTOR pathway is activated, resulting in the imbalance of Treg/Th17 and the local immune response of the middle ear.

\section{Discussion}

At present, the mechanism of immunity and allergy in OME pathogenesis is still controversial. Yellon et al. [11] first detected IL-1, IL-2, TNF-, INF-and IL-6 in middle ear exudate of children with chronic OME and found the incidence was $51 \%, 54 \%, 63 \%, 19 \%$ and $36 \%$, respectively. Doyle W J [12] found that I type such as allergy, Th2 cytokines and cell infiltration is likely to be the main cause of OME persist. Sobol et al. [13] found that Th2 cytokines IL-4, IL-5 and eosinophils were significantly higher than those with negative skin tests in the OME middle ear effusion with positive allergens.

Treg cell transcription factor is Foxp3, which mainly secretes cytokines such as TGF- and IL-10, and Treg cells participate in immune pathogenesis [14-16]. Th17 transcription factor is ROR T, mainly secreting IL17A-F and IL-6 series cytokines, which plays a physiological role in allergic and autoimmune diseases. IL-17 levels were significantly increased in rheumatoid arthritis, asthma and other immune diseases [15-16]. We found increased level of Th17 and inflammatory factor IL - 6 and reduced Treg cells factor TGF - beta and IL - 10 in OME.

In addition, ROR T/Foxp3 equilibrium also determines the direction of initial $\mathrm{T}$ cell differentiation toward Th17 or Treg cells [17]. In this study, the expression of ROR T was increased in PC group and decreased in NC group. However, Foxp3 expression was decreased in PC group and increased in NC group. In OME model group in middle ear mucosa, ROR gamma t has a strong positive expression and Foxp3 expression has a weak positivity, suggesting ROR gamma t/Foxp3 imbalance, further illustrating the whole body and middle ear local Treg/th17 balance in the process of occurrence and development of OME. Like other autoimmune diseases, middle ear mucosa as an extension of the upper respiratory tract mucous membrane, membrane layer of epithelium contains a lot of mucus glands, middle ear mucosa by antigen stimulation and called out ditto mucous membrane, organs become immune activity. Middle ear effuents in patients with OME with allergenic factors do not clear normally and may not be caused by initial viral/bacterial infection, but by circulating mast cells, eosinophil, etc., migrating to the site of inflammation to act on them and trigger a programmed immune response - "local allergic immune response" - to allergen stimulation [18].

HE staining of OME animal models showed that middle ear mucosa local buoyed by allergen, middle ear mucosa thickening, local hyperemia, tissue edema, glands expansion, plasma cells, eosinophil gathering, and the upper and lower respiratory tract after the allergen stimulation have the same immune response, middle ear organs become an immune activity. Regarding this, in the experiment of animal model of peripheral blood flow OME cytology, OME were also found in the rat model of spleen lymphocytes with negative correlation Treg and Th17 cells, so we speculated that the body state of sensitization may be easy to stimulate the important factors that cause middle ear mucosa, among them, the IL - 6 is a motivating factor with many biological activities. In the absence of IL-6, TGF- induces FOXP3 expression, which binds to ROR T and blocks its function, leading to the transformation of naive $\mathrm{T}$ cells into Treg. However, in the presence of IL-6, it eliminates the inhibition of FOXP3 on ROR t, leading to the conversion of naive t cells into Th17 cells [19]. This explains, on the one hand, the increased levels of IL-6 in middle ear effusion and peripheral blood in OME patients.

There are more and more evidence that on allergy treatment applied to OME aspects, this is different from the traditional upper respiratory tract (nose, sinuses, nasopharyngeal), located at the side of the respiratory tract (eustachian tube and middle ear tympanic cavity) allergic inflammation and also there are links, such as OME and the relationship between the AR epidemiology, anatomy, immunization and pathophysiology [20]

In addition, we verified the presence of PI3K/Akt/mTOR signaling pathway in OME, which affects $\mathrm{T}$ cell differentiation. Mammalian Target of rapamycin (mTOR) is a highly conserved serine/THR kinase that is activated and participates in the regulation of $\mathrm{T}$ cell growth, proliferation and differentiation under the action of glucocorticoids, nutrients (glucose, fatty acids, etc.) and various stressors [21]. 
An increasing number of studies have found that mTOR plays an important role in the development of a variety of autoimmune diseases by breaking the Thl7 / Treg balance, such as Systemic Lupus erythematosus (SLE) and rheumatoid Arthritis (RA). In the experimental autoimmune encephalomyelitis (EAE) mouse model, rapamycin was shown to inhibit Thl7 cell differentiation by blocking the mTOR/STAT3 signaling pathway [22].The Mtorc1 inhibitor rapamycin inhibits IL-17 and promotes Foxp3 expression, thereby selectively amplifying Treg cells and enhancing their stability and anti-inflammatory ability [23-24]. Compared with CON, OME middle ear mucosa had increased expression of PI3K/Akt/mTOR pathway marks, indicating activation of this signaling pathway, leading to inhibition of Treg cells differentiation and amplification, Treg/Th17 imbalance, and subsequent middle ear local immune response. However, the specific mechanism of Treg reduction in OME and its interaction with the TPI3K/Akt/mTOR signaling pathway remains to be further studied. These results indicate that the activation of PI3K/Akt/mTOR signaling pathway may partially inhibit the differentiation of Tregs, thus changing the Thl7 / Treg ratio.

This study has some limitations because of ethical limitations in obtaining middle ear mucosa and spleen tissue in OME patients and healthy individuals. Therefore, we established the OME rat model to compensate for local tissue detection and $\mathrm{T}$ cell detection in the spleen.

\section{Conclusion}

we found that the imbalance of the thl7 / Treg may be one of the important link of OME and the classic PI3K/Akt/mTOR signaling pathway may be involved in regulating $\mathrm{T}$ cell differentiation. Our study provides new insights into the etiological mechanisms of immunity and allergy with OME. In addition, mTOR inhibitors have been used to study the treatment of autoimmune diseases such as RA and SLE [25-26].Therefore, mTOR targeted therapy is expected to be a novel approach to treat OME in the future.

\section{References}

1. Bluestone CD,Klein J. Definitions,terminology and classification.In: Otitis Media in Infants and Children (Second Edition).Bluestone CD, Klein JO (Eds).WB Saunders Company, PA,USA,1-3 (1995).

2. Pau BC,Ng DK.Prevalence of otitis media with effusion in children with allergic rhinitis, a cross sectional study[J].Int J Pediatr Otorhinolaryngol,2016,84:156-160.doi: 10.1016 /j.ijporl.2016.03.008.

3. Chantzi FM,Kafetzis DA,Bairamis T,et al.IgE sensi- tization,respiratory allergy symptoms,and heritability independently increase the risk of otitis media with effusion[J].Allergy,2006,61(3):332-336.doi: 10.1111/j.1398-9995.2006.00971.x.

4. DENG Huiyi,YANG Qintai,WANG Weihao,et al.In- cidence of otitis media with effusion in adenoid hypertro- phy with allergic rhinitis in children[J].Journal of Sun Yat-Sen University(Medical Sciences),2017,38(1):101-105.doi:10.13471/j.cnki.j.Sun.yat-sen.univ ( med.sci).2017.0016.

5. Wright HD, Hurst D, Miotto D,et al. Increased expression of major basic protein and interleukin-5 in middle ear biopsy specimens from atopic patients with persistent otitis media with effusion.Otolaryngol Head Neck Surg,2000,123:533-538.

6. Jang CH,Kim YH.Characterization of cytolines present in pediatric otitis media with effusion:comparision of allergy positive and negative.Int J Pediatr Otorhinolaryngol,2002,66:37-40.

7. Nonaka M,Fukumoto A,Ozu C,et al.IL-5 and eotaxin levels in middle ear effusion and blood from asthmatics with otitis media with effusion.Acta Otolaryngol,2003,123:383-387.

8. Zhang L L.Effects of Yufeng Powder hesheng Decoction on Th17 / Treg cytokines in peripheral blood of patients with bronchial asthma[J].Journal of Modern Integrated Traditional Chinese and Western Medicine,2017,26( 28):3104-3106.

9. Xiao ling Zou,Zhuang gui Chen,Tian tuo Zhang,et al.Th17/Treg homeostasis,but not Th1/Th2 homeostasis is implicated in exacerbation of human bronchial asthma[J].Ther Clin Risk Manag,2018,14:16271636 .

10. Hardy SM,Heavner SB, White DR,et al.Late-phase allergy and eustachian tube dysfunction.Otolaryngol Head Neck Surg,2001,125(4):339-345. 
11. Yellon RF,Leonard G,Marucha P,etal.Demonstration of interleukin 6 in middle ear effusions.Arch 0tolaryngol Head Neck Surg,1992,118(7):745-748.

12. Doy le W J.The link between alle rgic rhinitis and otitis media.CurrOpin Alle rgy C lin Immuno 1,2002,2:21-25.

13. Sobol SE,Taha R,Schloss MD,et al.Th2 cytokine expression in atopic children with otitis media with effusion. J Allergy Clin Immunol,2002,110(1):125-130.

14. Wright HD, Hurst D, Miotto D,et al.Increased expression of major basic protein and interleukin-5 in middle ear biopsy specimens from atopic patients with persistent otitis media with effusion.Otolaryngol Head Neck Surg,2000,123:533-538.

15. Jang CH,Kim YH.Characterization of cytolines present in pediatric otitis media with effusion::comparision of allergy positive and negative.Int J Pediatr Otorhinolaryngol,2002,66:37-40.

16. Nonaka M,Fukumoto A,Ozu C,et al.IL-5 and eotaxin levels in middle ear effusion and blood from asthmatics with otitis media with effusion. Acta Otolaryngol,2003,123:383-387.

17. Xiao ling Zou,Zhuang gui Chen,Tian tuo Zhang,et al.Th17/Treg homeostasis, but not Th1/Th2 homeostasi,is implicated in exacerbation of human bronchial asthma[J].Ther Clin Risk Manag,2018,14:16271636.

18. YANG Qintai.Paying attention to the'lateral airway'allergic diseases in children[J].J Otolaryngol Ophthalmol Shandong Univ,2019,33( 1):59-62.

19. Kimura A,Kishimoto T.IL-6:regulator of Treg/Th17 balance.Eur J Immunol. 2010,40(7):1830-1835. doi: 10.1002/eji.201040391.

20. LI Ying,ZHAO Shouqin.Advances in clinical research on otitis media with effusion and allergic rhinitis in children[J].Chinese Journal of Otology,2016,14(2):295-29.

21. ZENG H,CHI H.mTOR signaling in the differentiation and function of regulatory and effector $\mathrm{T}$ cells[J].Curr Opin Immunol,2017,46:103-111.

22. CHEN Yc,LlAo F.CXCR3 signaling in glial cells ameliorates experimental autoimmune encephalomyelitis by restraining the generation of a pro-Thl7 cytokine milieu and reducing CNS-infihrating Th17 cells[J].J Neuroinflamm,2016,13(1):1-17.

23. LOPALCO G,LUCHERINl M,LOPALCO A,et al.Cytokine signatures in mucocutaneous and ocular Behcet's disease[J].Front Immunol,2017,8:200.

24. CHEN LC,NICHOLSON YT,ROSBOROUGH BR,et al.A Novel mTORCl-dependent.Akt-independent pathway differentiates the gut tropism of regulatory and conventional CD4 T cells[J].J Immunol,2016,197(4):1137-1147.

25. PERL A.Activation of mTOR(mechanistic target of rapamycin) in rheumatic diseases[J].Nat Rev Rheumatol,2016,12(3):169-182.

26. PERL A.mTOR activation is a biomarker and a central pathway to autoimmune disorders,cancer,obesity,and aging[J].Ann N YAcad Sci,2015,1346(1):33-44.Conflict of InterestThe authors confirm that there are no conflicts of interest.AcknowledgementThis work was supported by the Health science and Technology program of Shenzhen Longgang District(LGKCYLWS2019000227) and theShenzhen Key Medical Discipline Construction Fund(No.SZXK039).Ethics StatementThis animal experiments and the human studies informed consent of all participating subjects was obtained conform to internationally accepted standards and have been approved by the appropriate institutional review body. 

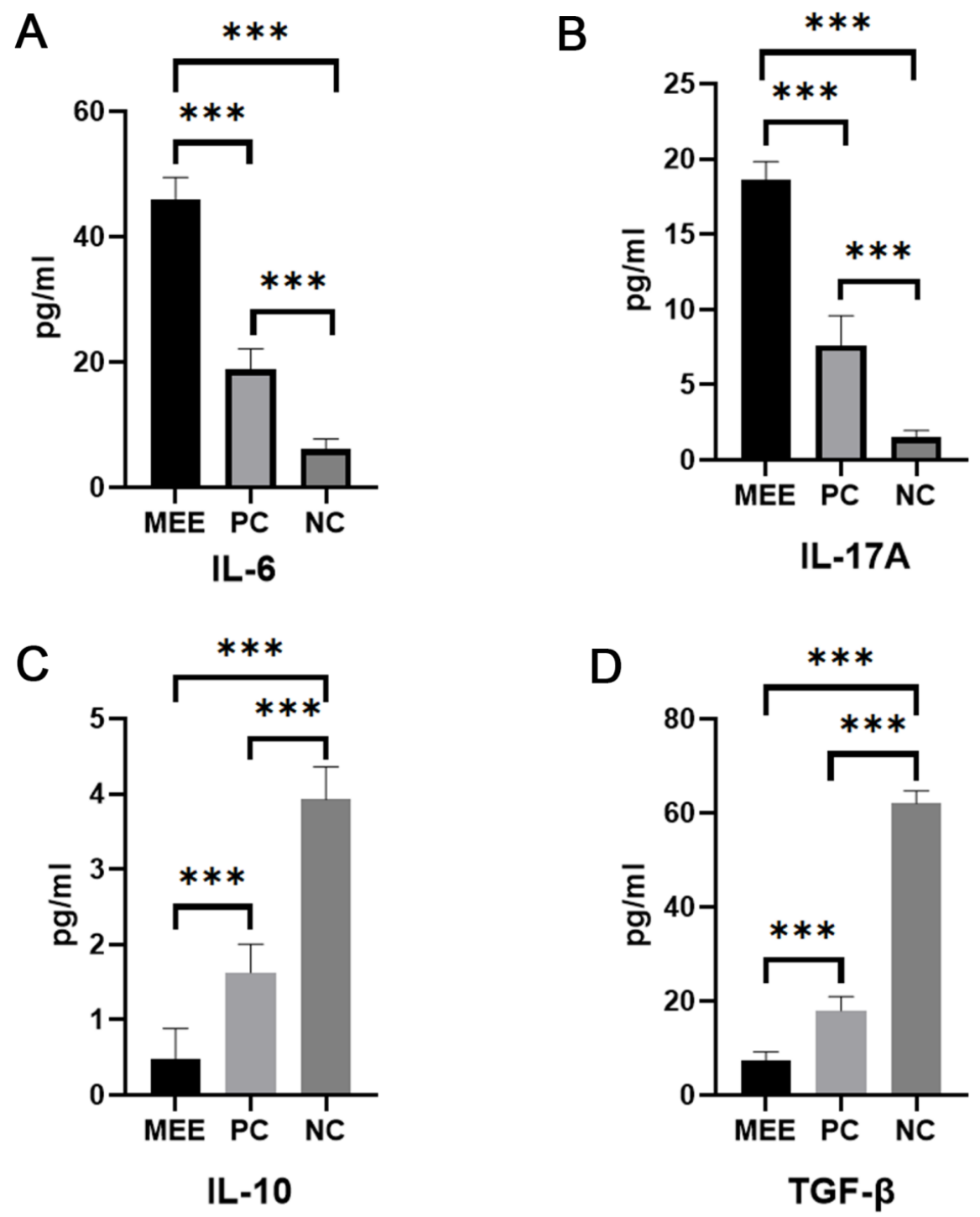

Figure 1 Detection of expression levels of target cytokines by middle ear effusion, peripheral blood serum of PC group, and peripheral blood serum of $\mathrm{NC}$ group A: expression level of IL-6;B: Expression level of IL-17A;C: Expression level of IL-10;D: TGF- expression level. $\left({ }^{*} \mathrm{P}<0.05,{ }^{* *} \mathrm{P}<0.01,{ }^{* * *} \mathrm{P}<0.001,{ }^{* * * *} \mathrm{P}<\right.$ $0.0001)$.

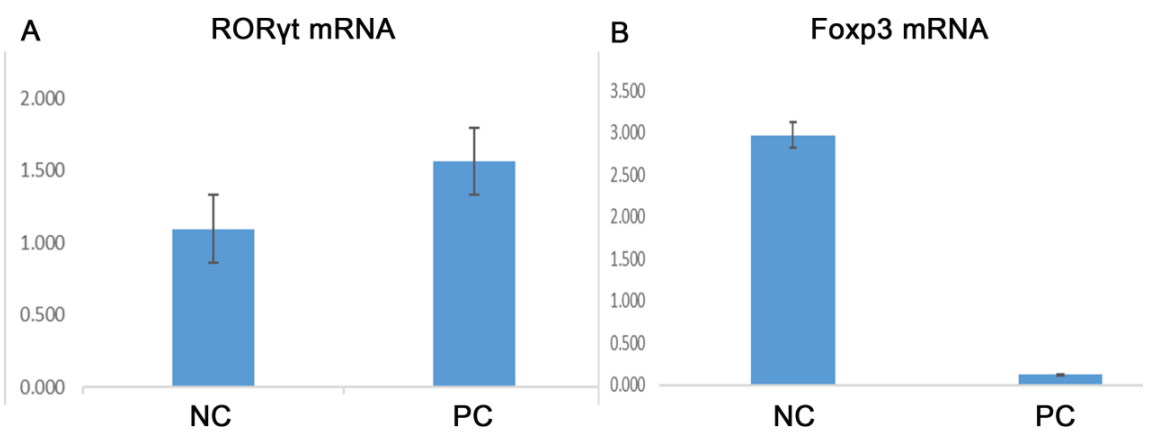


Figure 2 A:Expression of ROR $r$ mRNA, A specific transcription factor, in $\mathrm{PC}$ and $\mathrm{NC}$ peripheral blood.B:There were statistically significant differences in the expression of specific transcription factor FOXP3 mRNA between PC and NC peripheral blood $(\mathrm{P}<0.05)$.
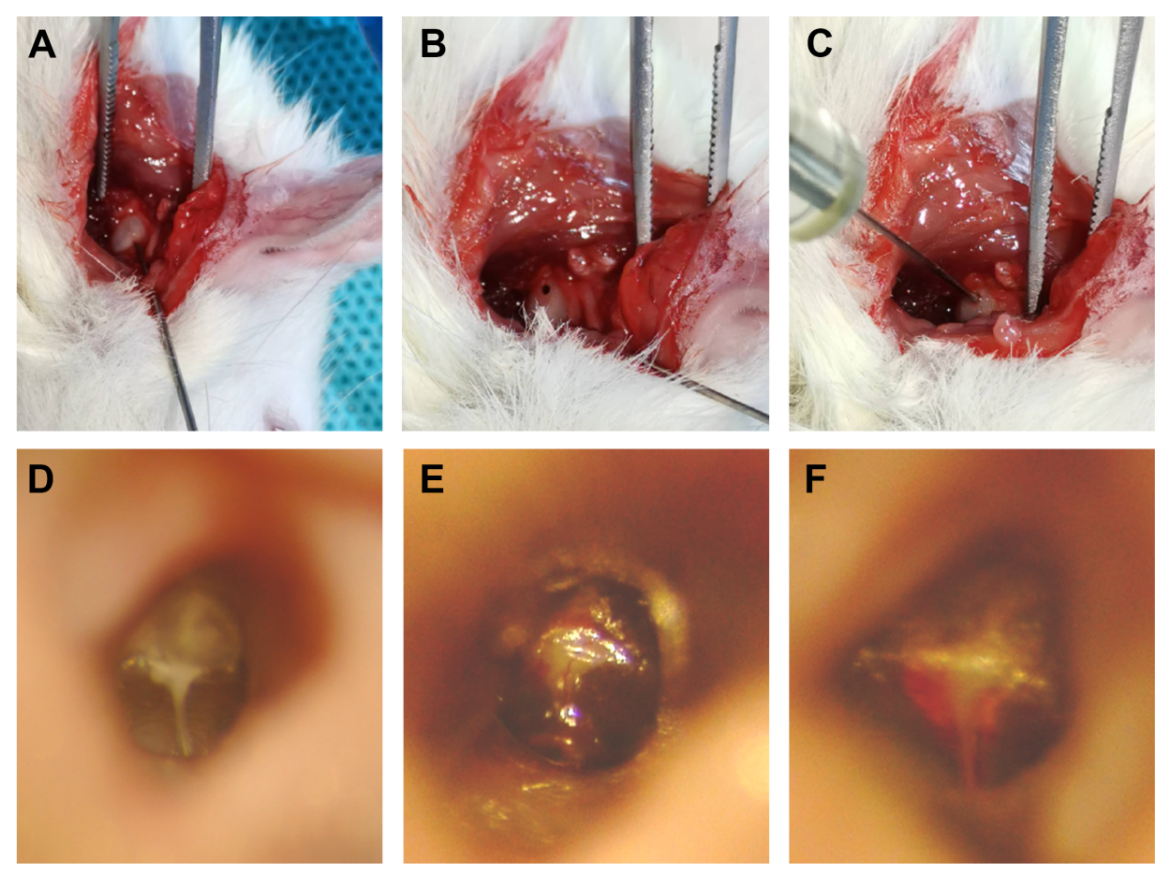

Figure 3 A: Exposing the auditory vesicle from the horizontal incision of hyoid bone; B: drilling the weakest part of the auditory vesicle; C: Injecting A sterile solution containing OVA $2 \mathrm{mg}$ into the hole with A microsyringe, and then sealing it off with A sterile gel sponge; D: Before modeling, observe the eardrum under A microscope for no hyperemia, swelling, or abnormalities in each mark;E: Acute congestion of the eardrum was observed under the microscope on the first day after modeling, and part of it was full.F: The congestion of tympanum was reduced, plump and orange under the microscope on the second day after modeling. 

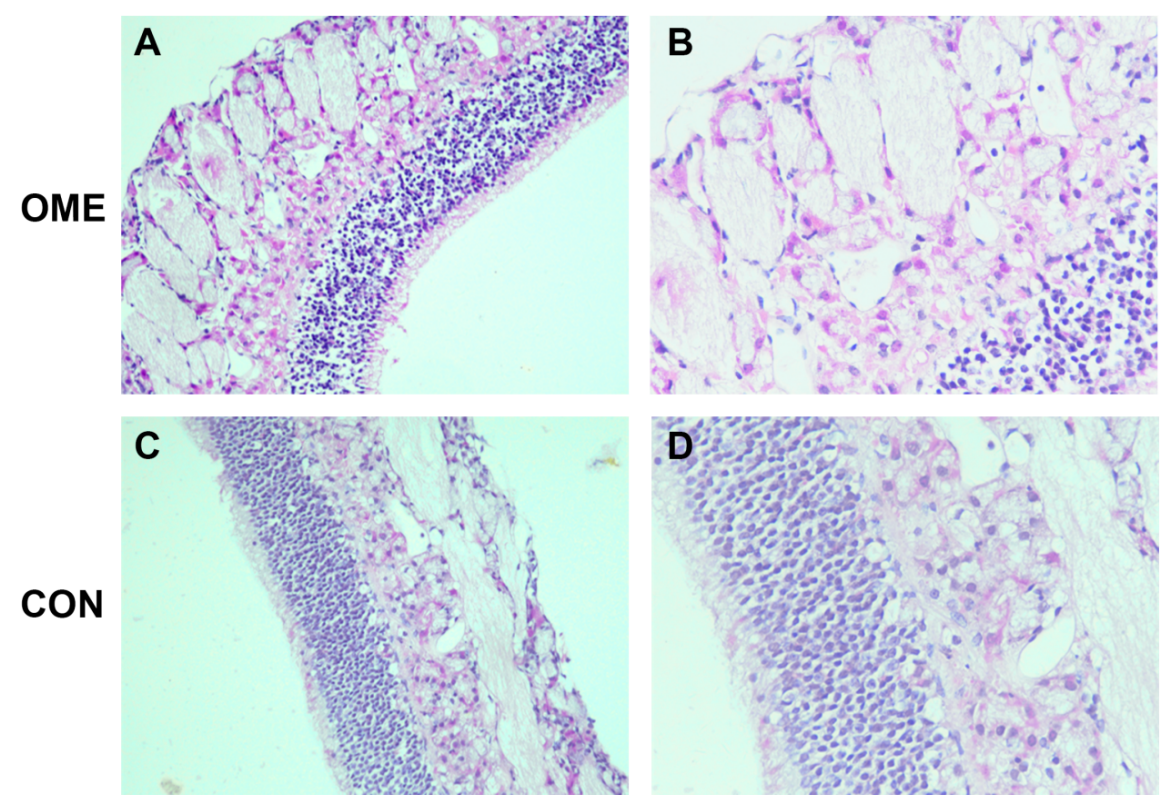

Figure $4 \mathrm{~A}$ : HE staining of the middle ear mucosa of the experimental group under 200x microscope; B: The middle ear mucosa of the experimental group was stained by HE under 400 times microscope.C: The middle ear mucosa of the control group was stained with HE under 200x microscope.D: The middle ear mucosa of the control group was stained by HE under 400 times microscope.

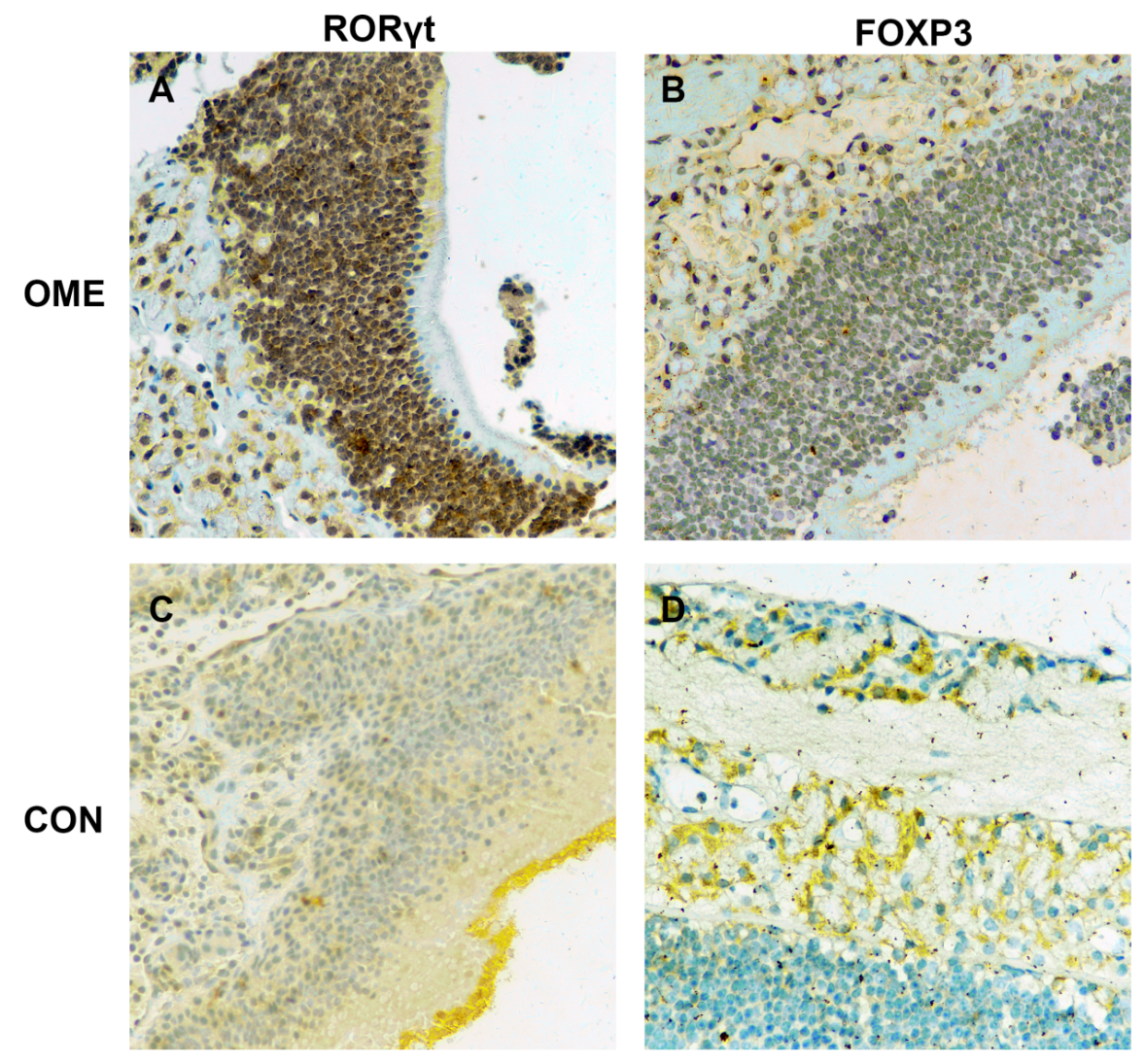


Figure 5 A: ROR $\gamma$ t expression in the experimental group;B: FOXP3 expression in the experimental group;C: ROR $\gamma \mathrm{T}$ expression in the control group;D: FOXP3 expression in the control group.
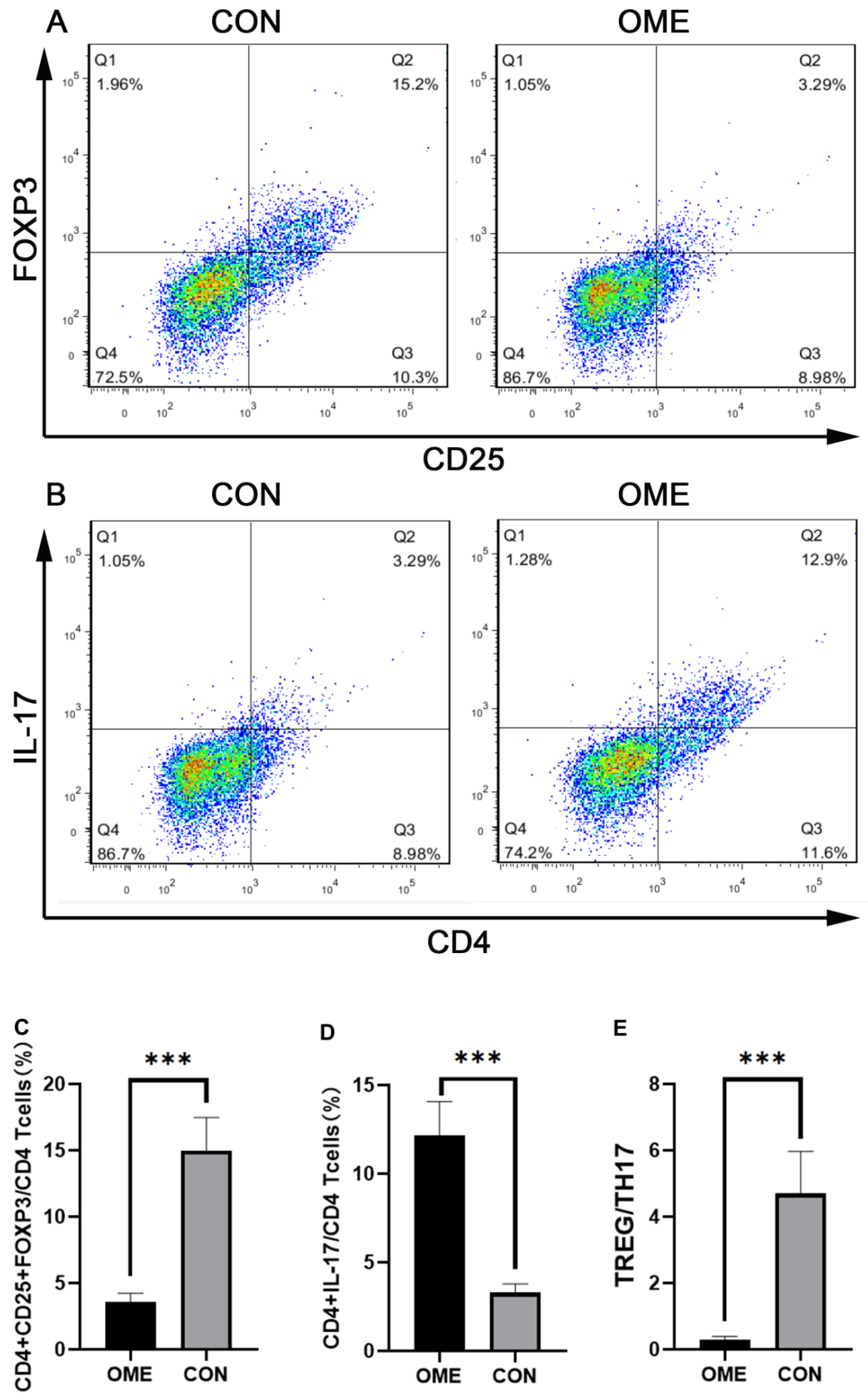

Figure 6 After successful modeling, CON and OME rats were sacrificed, spleen was collected, and Treg and Th17 cell frequencies in spleen were analyzed by FCM.A was the representative flow cytometry pattern 
of CD4, CD25 and FOXP3 staining of Treg cell frequencies in the spleen of CON and OME rats.B was the representative flow cytometry figure of CD3, CD4 and IL-17 staining of Th17 cells in CON and OME spleen.B was the typical flow cytometry figure of Th17 cells staining.C is the histogram of proportion of Treg cells to CD4+ in spleen of rats in the two groups.D is the histogram of the proportion of Th17 cells to CD4+ in spleen of rats in the two groups.E is a histogram of the ratio of Treg to Th17 cells in the spleens of the two groups of rats. $\left({ }^{*} \mathrm{P}<0.05,{ }^{* *} \mathrm{P}<0.01,{ }^{* * *} \mathrm{P}<0.001,{ }^{* * * *} \mathrm{P}<0.0001\right)$. 
A

OME CON

mTOR

p-mTOR

PI3K

p-PI3K

Akt

p-Akt

$\beta$-Actin 


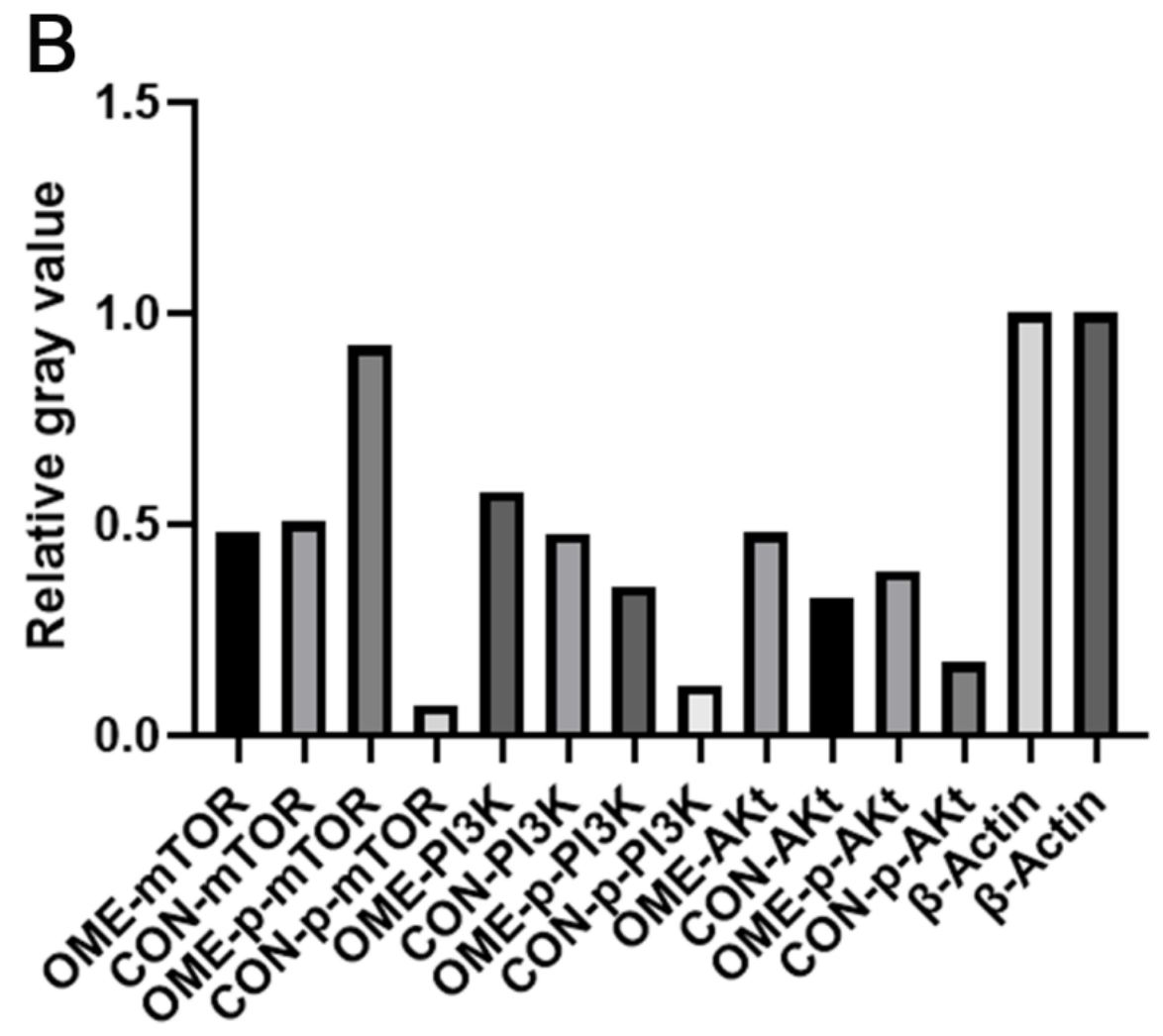

Figure 7 A: Expression of PI3K/Akt/mTOR pathway related proteins in the middle ear mucosa of the OME and CON groups;B: Relative gray values of PI3K/Akt/mTOR pathway related proteins in the middle ear mucosa of the OME and CON groups. 